\title{
FUNCTIONAL COHOMOLOGY OPERATIONS
}

\author{
BY \\ FRANKLIN P. PETERSON( ${ }^{(1)}$
}

1. Introduction. One of the aims of this paper is to investigate the following problem: if $f: X \rightarrow Y$ and $g: Y \rightarrow Z$ are nontrivial maps (in the sense of homotopy), then is the composition $g f: X \rightarrow Z$ nontrivial? Naively, we might ask the following question. Suppose $f$ is shown to be nontrivial by a functional primary cohomology operation, as defined by Steenrod [19], taking $y \in H^{*}(Y)$ into a nonzero element $x \in H^{*}(X)$; also suppose $g$ is similarly shown to be nontrivial by a functional primary cohomology operation taking $z \in H^{*}(Z)$ into $y$. Is the operation taking $z$ into $x$ a homotopy invariant of the composition $g f$ ? When the answer is "yes," then $g f$ is nontrivial. Our composition theorem (8.1) gives sufficient conditions for an answer of yes; under these conditions, the operation taking $z$ into $x$ is a functional "secondary" cohomology operation depending on $g f$.

Let $\theta: H^{n}(K ; \pi) \rightarrow H^{q}(K ; G)$ be a primary cohomology operation; i.e. $\theta$ is defined for all spaces $K$ and commutes with homomorphisms induced by continuous maps. Certain natural operations can be defined on the kernel of $\theta$; these are called secondary cohomology operations (for an example, see Adem $[1 ;$ p. 723]). When one of these is "zero," tertiary cohomology operations are defined, etc. In the first part of this paper, we make precise the notion of an $n$-ary cohomology operation and construct an exact couple in which the $\boldsymbol{n}$-ary cohomology operations appear as derivations. We also show that knowledge of certain of these operations is precisely what is needed for extension and classification problems.

In the second part of this paper, we give two equivalent definitions of functional $n$-ary cohomology operations. First, we generalize the method of Steenrod for defining functional primary cohomology operations [19], and second, we give a possibly more intuitive definition. The first definition is needed in order to make computations, while the second is needed in order to prove the composition theorem. The algebraic motivation for the generalization of Steenrod's method is quite simple and is as follows. Let $\left(A^{\prime}, C^{\prime}\right)$, $(A, C)$, and $\left(A^{\prime \prime}, C^{\prime \prime}\right)$ be exact couples, $i:\left(A^{\prime}, C^{\prime}\right) \rightarrow(A, C)$ and $j:(A, C)$ $\rightarrow\left(A^{\prime \prime}, C^{\prime \prime}\right)$ be couple maps, and let

$$
0 \rightarrow C^{\prime} \stackrel{i}{\rightarrow} C \stackrel{j}{\rightarrow} C^{\prime \prime} \rightarrow 0
$$

Received by the editors July 24, 1956.

(1) The author was a postdoctoral National Science Foundation fellow during the preparation of this paper. 
be exact. Then we have an exact triangle

$$
\begin{gathered}
C_{1}^{\prime} \stackrel{i_{1}}{\rightarrow} C_{1} \\
\Delta_{1} \nwarrow \swarrow j_{1} \\
C_{1}^{\prime \prime}
\end{gathered}
$$

where $\left(A_{n}, C_{n}\right)$ denotes the $n$th derived exact couple. If $\Delta_{1}=0$, then

$$
0 \rightarrow C_{1} \stackrel{i_{1}}{\rightarrow} C_{1} \stackrel{j_{1}}{\rightarrow} C_{1}^{\prime \prime} \rightarrow 0
$$

is exact and we may define $\Delta_{2}: C_{2}^{\prime \prime} \rightarrow C_{2}^{\prime}$. Continuing this process, we may define $\Delta_{n}: C_{n}^{\prime \prime} \rightarrow C_{n}^{\prime}$ when $\Delta_{n-1}=0, \cdots, \Delta_{1}=0$. Both the definitions and the proof of equivalence are completely algebraic; when they are applied to the exact couple constructed in the first part of the paper, they yield the functional $n$-ary cohomology operations; i.e. $\Delta_{n}$ becomes a functional $n$-ary cohomology operation.

After proving the composition theorem, we give some applications: a new proof of some results of Adem, and a generalization of those results modulo $p$. It is clear from these examples that in order to apply our technique to a particular case, one must calculate the cohomology groups of spaces having two, three, or more nonzero homotopy groups. In general, this is a difficult problem, and not very much is known.

Since our tools are all algebraic, we need certain sets of homotopy classes of maps to be groups. Thus, throughout the paper, we assume we are in the "stable range." Other higher order cohomology operations exist outside of this range; for a discussion of these, see the work of Massey [10]. The work of Massey and the aforementioned work of Adem motivated much of this paper.

2. The basic exact sequence. After recalling some definitions, we describe the basic exact sequence. The exact couple containing the higher order cohomology operations is constructed using this exact sequence.

Let $\pi(X ; Y)$ denote the set of homotopy classes of maps of $\left(X, x_{0}\right)$ into $\left(Y, y_{0}\right)$, where $x_{0} \in X, y_{0} \in Y$, and $Y$ is an arcwise connected space. This set has a distinguished element, called zero, represented by the constant map at $y_{0}$. The functor $\pi(X ; Y)$ is contravariant in the first variable and covariant in the second variable; i.e. maps $a:\left(X^{\prime}, x_{0}^{\prime}\right) \rightarrow\left(X, x_{0}\right)$ and $\phi:\left(Y, y_{0}\right) \rightarrow\left(Y^{\prime}, y_{0}^{\prime}\right)$ induce a function $\left(a^{\#}, \phi_{\#}\right): \pi(X ; Y) \rightarrow \pi\left(X^{\prime} ; Y^{\prime}\right)$ defined by composition. Let $S X$ denote the reduced suspension of $X$; i.e. $S X$ is defined by collapsing $X \times\{0\} \cup X \times\{1\} \cup\left\{x_{0}\right\} \times I \subset X \times I$ to a point $x_{0}$. Let $S^{r} X=S\left(S^{r-1} X\right)$. Let ${ }^{1} Y$ denote the space of loops in $Y$ at $y_{0}$. Let ${ }^{r} Y={ }^{1}\left({ }^{r-1} Y\right)$. This is a covariant functor on the category of all spaces and maps. It is well-known that $\pi(S X ; Y)$ is in a natural $1-1$ correspondence with $\pi\left(X ;{ }^{1} Y\right)$. It is also well-known that $\pi(X ; Y)$ has a natural group structure if $Y$ is a space of loops; the group struc- 
ture is abelian if $Y$ is a double space of loops [13, p. 261].

We recall the notion of an Eilenberg-MacLane space. Let $\pi$ be an abelian group and let $n$ be an integer $\geqq 1$. A space $X$ is an Eilenberg-MacLane space of type $K(\pi, n)$ if $\pi_{i}(X)=0$ for $i \neq n$ and $\pi_{n}(X)=\pi$. We denote such a space by $K(\pi, n)$.

Let us also recall the definition of a primary cohomology operation of type $(n, q ; \pi, G) . \theta$ is such a primary cohomology operation if $\theta: H^{n}(X ; \pi)$ $\rightarrow H^{q}(X ; G)$ is a function defined for all spaces $X$ such that if $f: X^{\prime} \rightarrow X$, then

$$
\begin{gathered}
H^{n}(X ; \pi) \stackrel{\theta}{\rightarrow} H^{q}(X ; G) \\
\downarrow f^{*} \quad \stackrel{\downarrow}{ } \quad \downarrow f^{*} \\
H^{n}\left(X^{\prime} ; \pi\right) \stackrel{\theta}{\rightarrow} H^{q}\left(X^{\prime} ; G\right)
\end{gathered}
$$

is a commutative diagram. One of the purposes of this paper is to generalize this notion. It is well-known that $\pi(X ; K(\pi, n)) \approx H^{n}(X ; \pi)$ if $X$ is a CWcomplex, and it follows that the set of primary cohomology operations of type $(n, q ; \pi, G)$ is in 1-1 correspondence with the elements of $H^{q}(\pi, n ; G)$ $=H^{q}(K(\pi, n) ; G)[16$, p. 220].

We say that

$$
\cdots \rightarrow Y_{n} \stackrel{\phi_{n}}{\rightarrow} Y_{n-1} \stackrel{\phi_{n-1}}{\rightarrow} Y_{n-2} \rightarrow \cdots
$$

is an exact sequence of spaces if the induced sequence

$$
\cdots \rightarrow \pi\left(X ; Y_{n}\right) \stackrel{\phi_{n}^{\sharp}}{\longrightarrow} \pi\left(X ; Y_{n-1}\right) \rightarrow \cdots
$$

is exact for all the $\mathrm{CW}$-complexes $X$ (the kernel of $\phi_{n^{\#}}$ is defined because $\pi\left(X ; Y_{n-1}\right)$ has a zero element $)$.

Let $p: E \rightarrow B$ be a fibre space in the sense of Serre [15]. Let $b_{0} \in B$ and $e_{0} \in F=p^{-1}\left(b_{0}\right) \subset E$ be base points for spaces of loops. Let $p_{1}: P \rightarrow B$ be the fibre space of paths in $B$ starting at $b_{0} . p_{1}^{-1}\left(b_{0}\right)={ }^{1} B$. A lifting of the map $p_{1}$ to a fibre preserving map $\tilde{p}: P \rightarrow E$ induces a map $i:{ }^{1} B \rightarrow F$.

Lemma 2.1. $\cdots \rightarrow^{r} F \stackrel{r}{j}_{\rightarrow}^{r} E \stackrel{r p}{\rightarrow}{ }^{r} B \stackrel{r-1}{\rightarrow}^{r-1} F \rightarrow \cdots \rightarrow \underset{\rightarrow}{p} \rightarrow B$ is an exact sequence of spaces. This exact sequence is natural with respect to fibre preserving maps.

Proof. Let $K$ be a CW-complex. Let $C K$ denote the cone on $K$ [18]. In a manner exactly analogous to constructing the exact homotopy sequence of a pair, one can show that

$$
\cdots \rightarrow \pi\left(S^{r} K ; F\right) \stackrel{j_{\sharp}}{\rightarrow} \pi\left(S^{r} K ; E\right) \stackrel{i_{*}}{\rightarrow} \pi\left(C S^{r-1} K, S^{r-1} K ; E, F\right) \stackrel{\partial_{1}}{\rightarrow} \pi\left(S^{r-1} K ; F\right) \rightarrow \cdots
$$


is an exact sequence [18]. Since $p: E \rightarrow B$ is a fibre space, $p^{\prime} t: \pi\left(C S^{r-1} K\right.$, $\left.S^{r-1} K ; E, F\right) \rightarrow \pi\left(S^{r} K ; B\right)$ is a $1-1$ correspondence and we have that

$$
\begin{aligned}
& \cdots \rightarrow \pi\left(S^{r} K ; F\right) \stackrel{j \leftrightarrow}{\rightarrow} \pi\left(S^{r} K ; E\right) \stackrel{p \leftrightarrow}{\rightarrow} \pi\left(S^{r} K ; B\right) \stackrel{\partial}{\rightarrow} \pi\left(S^{r-1} K ; F\right) \rightarrow \\
& \cdots \rightarrow \pi(K ; E) \stackrel{p \leftrightarrow}{\rightarrow} \pi(K ; B)
\end{aligned}
$$

is an exact sequence, where $\partial=\partial_{1}\left(p^{\prime} t\right)^{-1}$. Using the natural 1-1 correspondence $\pi(S K ; Y) \rightarrow \pi\left(K ;{ }^{1} Y\right)$ repeatedly, the lemma is proven.

3. Postnikov systems and the exact couple. We now give a brief resume of the definition and some of the basic properties of Postnikov systems (for details and proofs see [7] or [14]). Corresponding to such a system, we then construct an exact couple of spaces (in the obvious sense). The exact couple containing the higher order cohomology operations corresponding to the given Postnikov system is constructed by applying the functor $X \rightarrow \pi(K ; X)$ to this exact couple of spaces.

Let $\mathfrak{B}=\left(K(\pi, n), \theta ; X, \theta^{\prime} ; X^{\prime}, \theta^{\prime \prime} ; \cdots ; X^{(m-1)}, \theta^{(m)} ; \cdots\right)$ be a Postnikov system. This means that $\theta \in H^{q}(\pi, n ; G), \theta^{\prime} \in H^{q^{\prime}}\left(X ; G^{\prime}\right), \cdots, \theta^{(m)}$ $\in H^{z}\left(X^{(m-1)} ; G^{(m)}\right)$, where $z=q^{(m)}, \cdots$, that $\rho^{(m)}: X^{(m)} \rightarrow X^{(m-1)}$ is a fibre space with fibre $K\left(G^{(m)}, q^{(m)}-1\right)$ and $k$-invariant $\theta^{(m)}$, and that $q^{(m-1)}<q^{(m)}$. Hence $X^{(m)}$ has the following homotopy groups: $\pi$ in dimension $n, G$ in dimension $q-1, \cdots, G^{(m)}$ in dimension $q^{(m)}-1$. Furthermore,

$$
X^{(m)} \stackrel{\rho^{(m)}}{\longrightarrow} X^{(m-1)} \stackrel{\theta^{(m)}}{\longrightarrow} K\left(G^{(m)}, q^{(m)}\right)
$$

is an exact sequence of spaces, where the map $\theta^{(m)}: X^{(m-1)} \rightarrow K\left(G^{(m)}, q^{(m)}\right)$ is a representative of $\theta^{(m)} \in H^{z}\left(X^{(m-1)} ; G^{(m)}\right) \approx \pi\left(X^{(m-1)} ; K\left(G^{(m)}, q^{(m)}\right)\right)$, where $z=q^{(m)}$. To every space $X$, there corresponds a Postnikov system $\mathfrak{B}$ whose limit space $X^{(\infty)}$ is of the same homotopy type as $X$.

We need the following naturality property of Postnikov systems. Let $\phi:{ }^{\prime} \pi \rightarrow \pi, \phi_{m}:{ }^{\prime} G^{(m)} \rightarrow G^{(m)}$ be a sequence of homomorphisms. Corresponding to this sequence, one can define a new Postnikov system ' $\mathfrak{P}$ with homotopy groups ' $\pi$ in dimension $n, \cdots,{ }^{\prime} G^{(m)}$ in dimension $q^{(m)}-1, \cdots$ and a map $\Phi: ' \mathfrak{B} \rightarrow \mathfrak{B}$ which induces fibre preserving maps $\Phi_{m}:{ }^{\prime} X^{(m)} \rightarrow X^{(m)}$ inducing $\phi_{m}$ on the homotopy groups.

Corresponding to each fibre space $\rho^{(m)}: X^{(m)} \rightarrow X^{(m-1)}$, we construct, by Lemma 2.1, an exact sequence of spaces

$$
\begin{aligned}
& \cdots \rightarrow{ }^{r} X^{(m)} \stackrel{{ }^{r} \rho^{(m)}}{\longrightarrow} X^{(m-1)} \stackrel{r \theta^{(m)}}{\longrightarrow} K\left(G^{(m)}, q^{(m)}-r\right) \stackrel{r-1 \omega^{(m)}}{\longrightarrow}{ }^{r-1} X^{(m)} \rightarrow \\
& \cdots \rightarrow X^{(m)} \stackrel{\rho^{(m)}}{\longrightarrow} X^{(m-1)} \stackrel{\theta^{(m)}}{\longrightarrow} K\left(\mathcal{I}^{(m)}, q^{(m)}\right) .
\end{aligned}
$$

These exact sequences of spaces fit together to form an exact couple of spaces as in figure 1. 


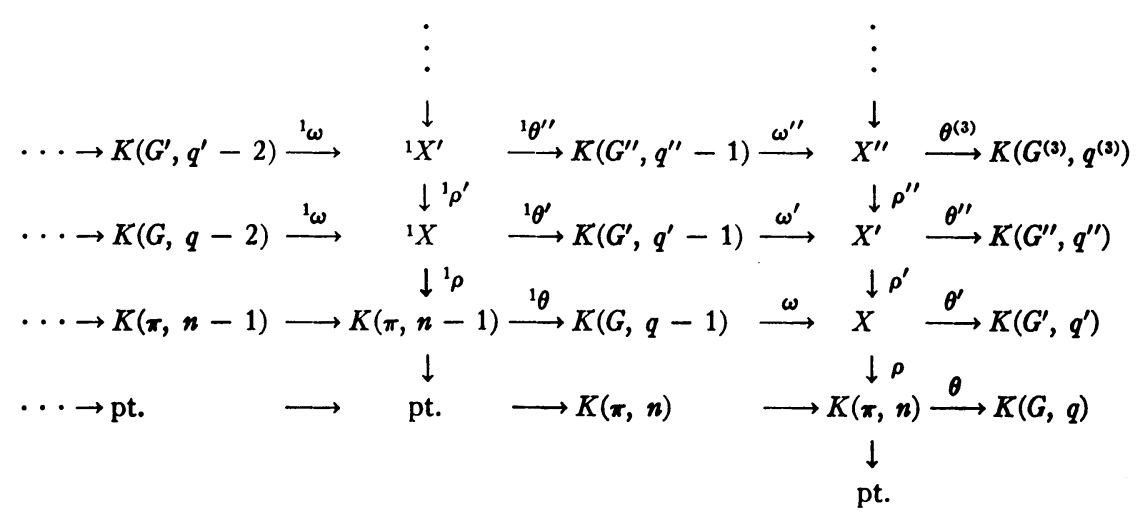

FIGURE 1

We now make an assumption to guarantee that all the sets of homotopy classes of maps which we will study have a natural group structure. We assume throughout the rest of the paper that $X^{(\infty)}$ is a space of loops. This assumption is equivalent to assuming that each $k$-invariant $\theta^{(m)}$ is a suspension, in the sense that $\theta^{(m)}={ }^{1} \psi^{(m)}$ for some $\psi^{(m)}$ (a special case of this statement is proved in [20]; the general case follows similarly). Hence our assumption implies that each $X^{(m)}$ is a space of loops and thus that $\pi\left(K ; X^{(m)}\right)$ is a group.

Applying the functor $X \rightarrow \pi(K ; X)$ to each space in the exact couple of spaces of Figure 1, we get the exact couple corresponding to the given Postnikov system $\mathfrak{P}$ (see [8] for definition and properties of exact couples). For notational sake, we define

$$
\begin{aligned}
& C^{r, s}=H^{z}\left(K ; G^{(r-1)}\right), \\
& A^{r, s}=\pi\left(K ;{ }^{-s} X^{(r-1)}\right)
\end{aligned}
$$

where $z=q^{(r-1)}+s-1, G^{(-1)}=\pi, q^{(-1)}=n+1, X^{(-1)}=K(\pi, n), X^{(p)}=$ pt. if $p<-1$, and $G^{(p)}=0$ if $p<-1$. Also,

$$
\begin{aligned}
f^{r, s}: A^{r, s} & \rightarrow A^{r-1, s}, \\
g^{r-1, s}: A^{r-1,8} & \rightarrow C^{r, s+1,},
\end{aligned}
$$

and

$$
h^{r, s}: C^{r, s} \rightarrow A^{r, 8}
$$

are induced by ${ }^{-s} \rho^{(r-1)},-8 \theta^{(r-1)}$, and ${ }^{-s} \omega^{(r-1)}$ respectively. Note that our assumption implies that $A^{r, 8}$ is a group, that $C^{r, s}$ is an abelian group, and that $f^{r, s}, g^{r, s}$, and $h^{r, s}$ are homomorphisms. The constructions of [8] can be carried out if $A^{r, s}$ is not necessarily abelian.

4. Higher order cohomology operations. In this section, we give the definition of higher order cohomology operations and state their main prop- 
erties. Motivation for this definition arises from the well-known concept of a primary cohomology operation, from the known examples of "secondary cohomology operations" (e.g., [1, p. 723], [10], or [17]), from our remarks relating the definition to cohomotopy groups, and from the heuristic feeling that $n$-ary cohomology operations should be defined on the kernel of an $(n-1)$-ary cohomology operation with values in the cokernel of an $(n-1)$-ary cohomology operation (see [9, p. 343]). Our attention is centered on higher order operations defined on the $C^{0,0}$ term of the above exact couple; we prove that the operations on $C^{r, s}$ are equivalent to such basic operations.

Let $\left(A_{n}, C_{n} ; f_{n}, g_{n}, h_{n}\right)$ denote the $n$th derived exact couple of the exact couple $(A, C)$ defined in $\S 3 . f_{n}$ has bidegree $(-1,0), g_{n}$ has bidegree $(n+1,1)$, $h_{n}$ has bidegree $(0,0)$, and hence $d_{n}=g_{n} h_{n}$ has bidegree $(n+1,1)$. We define the $n$-ary cohomology operation corresponding to the given Postnikov system $\mathfrak{B}$ to be $\left(\theta, \theta^{\prime}, \cdots, \theta^{(n-1)}\right)=d_{n-1}^{0,0}: C_{n-1}^{0,0} \rightarrow C_{n-1}^{n, 1}$. It is immediate that this operation is natural with respect to maps $a: L \rightarrow K$; i.e. $a^{*} d_{n-1}^{0,0}=d_{n-1}^{0,0} a^{*}: C(K)_{n-1}^{0,0}$ $\rightarrow C(L)_{n-1}^{n, 1}$.

When $n=1$, we get the notion of a primary cohomology operation $\theta=d^{0,0}: H^{n}(K ; \pi) \rightarrow H^{q}(K ; G)$. As another example, we mention the following well-known secondary operation of Adem [1, p. 723]. Let $\theta=S q^{2}: H^{n}(K ; Z)$ $\rightarrow H^{n+2}\left(K ; Z_{2}\right)$. Let $\rho: X \rightarrow K(Z, n)$ be the fibre space with $k$-invariant $\theta$ and fibre $K\left(Z_{2}, n+1\right)$. Let $\theta^{\prime} \in H^{n+3}\left(X ; Z_{2}\right)$ be the element corresponding to $S q^{2}$ in the fibre. Then $\left(\theta, \theta^{\prime}\right)$ is Adem's operation $\Phi$. Further examples will be discussed in more detail in $\$ 9$.

We now show that the $d_{n-1}^{r, s}$ in the exact couple $(A, C)$ are essentially $n$-ary cohomology operations corresponding to a different Postnikov system. Corresponding to the given system $\mathfrak{B}$ and the sequence of homomorphisms $\phi: 0 \rightarrow \pi, \cdots, \phi_{r-2}: 0 \rightarrow G^{(r-2)}, \phi_{r-1}: G^{(r-1)} \rightarrow G^{(r-1)}$ the identity, $\phi_{r}: G^{(r)} \rightarrow G^{(r)}$ the identity, $\cdots$, we can define a new Postnikov system ' $\mathfrak{P}$ and a map $\Phi: ' \mathfrak{B} \rightarrow \mathfrak{B}$. If we denote the corresponding exact couple by $\left({ }^{\prime} A,{ }^{\prime} C\right)$, we have a couple map $\phi:\left({ }^{\prime} A,{ }^{\prime} C\right) \rightarrow(A, C)$ of bidegree $(r, s)$. It follows immediately from our construction of $\left({ }^{\prime} A,{ }^{\prime} C\right)$ that $\phi^{k, l}:{ }^{\prime} C^{k, l} \rightarrow C^{k+r, l+s}$ is an isomorphism if $k \geqq 0$. Hence, $\phi_{n}^{k, l}:{ }^{k, l} \rightarrow C_{n}^{k+r, l+s}$ is an isomorphism if $k \geqq n$ and is onto if $k \geqq 0$. Also, $\phi_{n-1}^{n, 1} d_{n-1}^{0,0}=d_{n-1}^{r, s} \phi_{n-1}^{0,0}$. Since $\phi_{n-1}^{n, 1}$ is an isomorphism and $\phi_{n-1}^{0,0}$ is onto, $d_{n-1}^{r, s}$ is given by ' $d_{n-1}^{0,0}$.

It follows from the preceding paragraph that the $n$-ary cohomology operation $d_{n-1}^{0,0}$ is defined on the kernel of the $(n-1)$-ary cohomology operation $d_{n-2}^{0,0}$ with values in a subgroup of the cokernel of the $(n-1)$-ary cohomology operation $d_{n-2}^{1,0}$.

The original motivation for our study of higher order cohomology operations came from examining the first derived cohomotopy exact couple (see $[12$, p. 248] or [8]). It can be shown that there is a couple map from the first derived cohomotopy exact couple studied in [8] to the exact couple defined above which is an isomorphism. This fact is not needed in this paper, so we 
will not prove it here. Also, there is a couple map from the cohomotopy exact couple studied in [8] to the cohomotopy exact couple studied in [12] which is an isomorphism on the " $C$ "-groups and hence an isomorphism on the associated spectral sequences. Hence the operations arising in the first derived cohomotopy exact couple are higher order cohomology operations in the sense defined above.

5. Functional cohomology operations: generalization of the Steenrod method. We will give two methods for defining functional higher order cohomology operations and then prove equivalence of the two methods. The first method generalizes the definition of Steenrod for functional primary cohomology operations: let us recall that definition [19].

Let $a: L \rightarrow K$. Let $M=K \bigcup_{a} C L$, where $C L$ denotes the cone on $L$, and $C L$ is attached to $K$ by $a$. Let $b: K \rightarrow M$ be the inclusion, and $c: M \rightarrow S L$ be defined by collapsing $K$ to a point. The sequence

$$
L \stackrel{a}{\rightarrow} K \stackrel{b}{\rightarrow} M \stackrel{c}{\rightarrow} S L \stackrel{S a}{\rightarrow} S K \rightarrow \cdots \rightarrow S^{r} K \stackrel{S^{r} b}{\longrightarrow} S^{r} M \stackrel{S^{r} c}{\longrightarrow} S^{r+1} L \rightarrow \cdots
$$

is a coexact sequence of spaces in the sense that the induced sequence

$$
\begin{aligned}
& \cdots \rightarrow \pi\left(S^{r+1} L ; Y\right) \stackrel{S^{r} c^{t}}{\rightarrow} \pi\left(S^{r} M ; Y\right) \stackrel{S^{r} b t}{\longrightarrow} \pi\left(S^{r} K ; Y\right) \rightarrow \\
& \cdots \rightarrow \pi(M ; Y) \stackrel{b^{f}}{\rightarrow} \pi(K ; Y) \stackrel{a^{\prime}}{\rightarrow} \pi(L ; Y)
\end{aligned}
$$

is exact for every $Y[4$, p. 81$]$. Let $\theta: K(\pi, n) \rightarrow K(G, q)$ be a given additive cohomology operation. Then we have a commutative diagram:

$$
\begin{aligned}
& H^{n-1}(K ; \pi) \stackrel{a^{*}}{\rightarrow} H^{n-1}(L ; \pi) \stackrel{c^{*}}{\rightarrow} H^{n}(M ; \pi) \stackrel{b^{*}}{\rightarrow} H^{n}(K ; \pi) \stackrel{a^{*}}{\rightarrow} H^{n}(L ; \pi)
\end{aligned}
$$

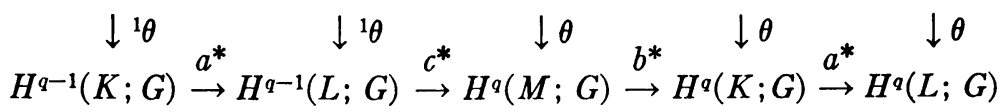

(using the fact that $\pi(S K ; K(\pi, n)) \approx \pi\left(K ;{ }^{1} K(\pi, n)\right) \approx \pi(K ; K(\pi, n-1)$ ) $\left.\approx H^{n-1}(K ; \pi)\right)$. Let $u \in H^{n}(K ; \pi)$ be such that $a^{*}(u)=0$ and $\theta(u)=0$. Define $\theta_{a}(u) \in H^{a-1}(L ; G) / \operatorname{Im} a^{*}+\operatorname{Im}^{1} \theta$ by $\theta_{a}(u)=\left(c^{*}\right)^{-1} \theta\left(b^{*}\right)^{-1}(u)$. This is essentially the definition of Steenrod.

We now generalize the definition of Steenrod to functional higher order cohomology operations. We first give the algebraic formalism.

Let $\left(A^{\prime}, C^{\prime}\right),(A, C)$, and $\left(A^{\prime \prime}, C^{\prime \prime}\right)$ be bigraded exact couples, with $f: A \rightarrow A$ of bidegree $(-1,0), g: A \rightarrow C$ of bidegree $(1,1)$, and $h: C \rightarrow A$ of bidegree $(0,0)$. Let $i:\left(A^{\prime}, C^{\prime}\right) \rightarrow(A, C)$ and $j:(A, C) \rightarrow\left(A^{\prime \prime}, C^{\prime \prime}\right)$ be couple maps of bidegree $(0,1)$ and $(0,0)$ respectively. Assume

$$
0 \rightarrow\left(A^{\prime}, C^{\prime}\right) \stackrel{i}{\rightarrow}(A, C) \stackrel{j}{\rightarrow}\left(A^{\prime \prime}, C^{\prime \prime}\right) \rightarrow 0
$$

is exact in the sense that 


$$
0 \rightarrow C^{\prime} \stackrel{i}{\rightarrow} C \stackrel{j}{\rightarrow} C^{\prime \prime} \rightarrow 0
$$

is exact. By the usual algebraic construction, we get an exact triangle [6, p. 54],

$$
\begin{gathered}
C_{1}^{\prime} \stackrel{i_{1}}{\rightarrow} C_{1} \\
\Delta_{1} \nwarrow \swarrow j_{1} \\
C_{1}^{\prime \prime}
\end{gathered}
$$

where $\Delta_{1}(\{u\})=(i)^{-1} d(j)^{-1}(u)$ for $u \in C^{\prime \prime}$ representing $\{u\} \in C_{1}^{\prime \prime}$, and $\Delta_{1}$ is of bidegree $(1,0)$. If $\Delta_{1}=0$, then

$$
0 \rightarrow\left(A_{1}^{\prime}, C_{1}^{\prime}\right) \stackrel{i_{1}}{\rightarrow}\left(A_{1}, C_{1}\right) \stackrel{j_{1}}{\rightarrow}\left(\left(A_{1}^{\prime \prime}, C_{1}^{\prime}\right) \rightarrow 0\right.
$$

is exact in the above sense and we may repeat the process; i.e.

$$
\begin{aligned}
& C_{2}^{\prime} \stackrel{i_{2}}{\rightarrow} C_{2} \\
& \Delta_{2} \nwarrow \swarrow j_{2}
\end{aligned}
$$

$C_{2}^{\prime \prime}$

is an exact triangle. In general, if $\Delta_{1}=0, \cdots, \Delta_{n-1}=0$, then we may define $\Delta_{n}: C_{n}^{\prime \prime} \rightarrow C_{n}^{\prime}$ of bidegree $(n, 0)$ such that

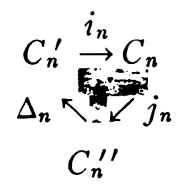

is an exact triangle. The formula for $\Delta_{n}$ is given by

$$
\Delta_{n}(\{u\})=(i)^{-1} g(f)^{-n+1} h(j)^{-1}(u) .
$$

Let $\phi$ be a homomorphism of exact sequences of exact couples; i.e.

$$
\begin{aligned}
& 0 \rightarrow\left(A^{\prime}, C^{\prime}\right) \stackrel{i}{\rightarrow}(A, C) \stackrel{j}{\rightarrow}\left(A^{\prime \prime}, C^{\prime \prime}\right) \rightarrow 0 \\
& \downarrow \phi^{\prime} \quad \downarrow \phi \quad \downarrow \phi^{\prime \prime} \\
& 0 \rightarrow\left({ }^{\prime} A^{\prime},{ }^{\prime} C^{\prime}\right) \stackrel{{ }^{\prime} i}{\rightarrow}\left({ }^{\prime} A,{ }^{\prime} C\right) \stackrel{{ }^{\prime} j}{\rightarrow}\left({ }^{\prime} A^{\prime \prime},{ }^{\prime} C^{\prime \prime}\right) \rightarrow 0
\end{aligned}
$$

is a commutative diagram, where $\phi^{\prime}, \phi$, and $\phi^{\prime \prime}$ are couple maps. Then ${ }^{\prime} \Delta_{n} \phi^{\prime \prime}=\phi^{\prime} \Delta_{n}: C_{n}^{\prime \prime} \rightarrow^{\prime} C_{n}^{\prime}$, when defined. Hence our definition is natural.

Given an exact couple of spaces corresponding to a given Postnikov system $\mathfrak{B}$, as in Figure 1. Applying this functor to the coexact sequence of spaces 


$$
L \stackrel{a}{\rightarrow} K \stackrel{b}{\rightarrow} M \stackrel{c}{\rightarrow} S L \stackrel{S a}{\rightarrow} S K \rightarrow \cdots
$$

we get an exact sequence of exact couples. Let $\left(A^{\prime}, C^{\prime}\right)$ be the exact couple for $L,(A, C)$ the exact couple for $M,\left(A^{\prime \prime}, C^{\prime \prime}\right)$ the exact couple for $K, i=c^{*}$, and $j=b^{*}$. If $a^{*}: H^{*}(K ; G) \rightarrow H^{*}(L ; G)$ is zero for all coefficient groups $G$, then the hypotheses of the above algebraic formalism are satisfied. We define the functional $n$-ary cohomology operation corresponding to the given Postnikov system $\mathfrak{P}$ to be

$$
\left(\theta, \theta^{\prime}, \cdots, \theta^{(n-1)}\right)_{a}=\Delta_{n}^{0,0} .
$$

If $\phi$ is a map of one exact couple of spaces into another exact couple of spaces, then we get an induced map $\phi_{\sharp}$ on the exact couples which commutes with $\Delta_{n}^{p, q}$.

6. Functional cohomology operations: the second method. In this section, we give a second definition of functional higher order cohomology operations. This definition is needed in order to prove our composition theorem ( $\$ 8$ ). Unfortunately, this definition can't be given in as invariant a manner as the first definition; however, it is a more intuitive definition.

We first give the necessary algebraic formalism. Let $\left(A^{\prime \prime}, C^{\prime \prime}\right)$ be an exact couple. $u \in C^{\prime \prime}$ can be lifted $n$-stages if there exists an element $a \in A^{\prime \prime}$ such that $h^{\prime \prime}(u)=\left(f^{\prime \prime}\right)^{n}(a)$. It is easily seen that $u$ can be lifted $n$-stages if and only if $d(u)=0, d_{1}(\{u\})=0, \cdots, d_{n-1}(\{u\})=0$. Furthermore, for a given $u, a \in A^{\prime \prime}$ is unique modulo the images of $h^{\prime \prime},\left(f^{\prime \prime}\right)^{-1} h^{\prime \prime}, \cdots,\left(f^{\prime \prime}\right)^{-n+1} h^{\prime \prime}$.

Let $k:\left(A^{\prime \prime}, C^{\prime \prime}\right) \rightarrow\left(A^{\prime \prime \prime}, C^{\prime \prime \prime}\right)$ be a couple map. We assume that $k: C^{\prime \prime} \rightarrow C^{\prime \prime \prime}$ is zero. For convenience, we set $\bar{C}^{\prime \prime \prime}=C^{\prime \prime \prime} / \mathrm{Im} g^{\prime \prime \prime}$. We now define a sequence of operations, $d_{n, k}$.

Define

$$
d_{1, k}: C_{1}^{\prime \prime} \rightarrow \bar{C}^{\prime \prime \prime}
$$

as follows: Let $u \in C^{\prime \prime}$ represent $\{u\} \in C_{1}^{\prime \prime}$. Let $a \in A^{\prime \prime}$ be a lifting of $u$ one stage. Then $f^{\prime \prime \prime} k(a)=k f^{\prime \prime}(a)=k h^{\prime \prime}(u)=h^{\prime \prime \prime} k(u)=0$. Hence we may define $d_{1, k}(\{u\})=\left(h^{\prime \prime \prime}\right)^{-1} k(a)$. This is well-defined because changing $a$ by $\operatorname{Im} h^{\prime \prime}$ changes $d_{1, k}(\{u\})$ by $\operatorname{Im}\left(\left(h^{\prime \prime \prime}\right)^{-1} k h^{\prime \prime}\right)=\operatorname{Im}\left(\left(h^{\prime \prime \prime}\right)^{-1} h^{\prime \prime \prime} k\right)=0$.

In general, if $d_{n-1, k}, \cdots, d_{1, k}$ are zero, we define for $n>1$

$$
d_{n, k}: C_{n}^{\prime \prime} \rightarrow \bar{C}^{\prime \prime \prime}
$$

as follows: Let $a \in A^{\prime \prime}$ be a lifting of $u n$-stages. Then $f^{\prime \prime \prime} k(a)=k f^{\prime \prime}(a)$ $=h^{\prime \prime \prime} d_{n-1, k}(\{u\})=0$. Hence we may define $d_{n, k}(\{u\})=\left(h^{\prime \prime \prime}\right)^{-1} k(a) \in \bar{C}^{\prime \prime \prime}$. This is well-defined because changing $a$ by $\operatorname{Im} h^{\prime \prime}, \operatorname{Im}\left(\left(f^{\prime \prime}\right)^{-1} h^{\prime \prime}\right), \cdots$, $\operatorname{Im}\left(\left(f^{\prime \prime}\right)^{-n} h^{\prime \prime}\right)$ changes $d_{n, k}(\{u\})$ by $0, \operatorname{Im} d_{1, k}, \cdots, \operatorname{Im} d_{n-1, k}$, respectively, and these are all assumed to be zero. 


$$
\begin{aligned}
& \left(A^{\prime \prime}, C^{\prime \prime}\right) \stackrel{k}{\rightarrow}\left(A^{\prime \prime \prime}, C^{\prime \prime \prime}\right) \\
& \begin{array}{c}
\downarrow \phi^{\prime \prime} \\
\left({ }^{\prime} A^{\prime \prime},{ }^{\prime} C^{\prime \prime}\right) \stackrel{\downarrow}{\downarrow} \stackrel{\downarrow \phi^{\prime \prime \prime}}{\rightarrow}\left({ }^{\prime} A^{\prime \prime \prime},{ }^{\prime} C^{\prime \prime \prime}\right)
\end{array}
\end{aligned}
$$

is a commutative diagram, where $\phi^{\prime \prime}, \phi^{\prime \prime \prime}, k$, and ' $k$ are couple maps, then ${ }^{\prime} d_{n, ' k} \phi^{\prime \prime}=\phi^{\prime \prime \prime} d_{n, k}: C_{n}^{\prime \prime} \rightarrow \bar{C}^{\prime \prime \prime}$ when defined. Hence our definition is natural.

As in $\$ 5$, the exact couples in the applications are bigraded. Assuming $k$ has bidegree $(0,0), f, g$, and $h$ as before, then $d_{n, k}$ has bidegree $(n, 0)$; i.e. $d_{n, k}^{p, q}: C_{n}^{\prime \prime p, q} \rightarrow \bar{C}^{\prime \prime \prime p+n, q}$. Furthermore, in order to define $d_{n, k}^{p, q}$, it is only necessary to assume $d_{n-1, k}^{p, q}, d_{n-1, k}^{p+1, q}, d_{n-2, k}^{p, q}, \cdots, d_{n-2, k}^{p+2, q}, \cdots, d_{1, k}^{p, q}, \cdots, d_{1, k}^{p+n-1, q}$ are zero.

We apply our algebraic formalism to the same situation as in $\S 5$. Let $\left(A^{\prime \prime}, C^{\prime \prime}\right)$ be the exact couple for $K,\left(A^{\prime \prime \prime}, C^{\prime \prime \prime}\right)$ be the exact couple for $L$, and let $k=a^{*}$. Our second definition of the functional $n$-ary cohomology operation corresponding to the given Postnikov system $\mathfrak{B}$ is

$$
\left(\theta, \theta^{\prime}, \cdots, \theta^{(n-1)}\right)_{a}=d_{n, k}^{0,0}
$$

If $\phi$ is a map of one exact couple of spaces into another exact couple of spaces, then we get an induced map $\phi \#$ on the exact couple which commutes with $d_{n, k}^{p, n}$. The following two naturality properties also follow immediately from the above remarks.

TheOREM 6.1. Let $b: M \rightarrow L$ and $a: L \rightarrow K$. Then $b^{*} d_{n, a}^{p, q}=d_{n, a b}^{p, q}$ when defined.

Theorem 6.2. Let $a: L \rightarrow K$ and $b: K \rightarrow J$. Then $d_{n, a}^{p, q} b^{*}=d_{n, b a}^{p, q}$ when defined.

7. Equivalence of the two methods and an application to homotopy theory. We now prove that our two methods of defining functional $n$-ary cohomology operations are equivalent. As in $\$ \S 5$ and 6, we first give the algebraic formalism. Using the first method, we then show that functional $n$-ary cohomology operations are essentially $n$-ary cohomology operations in the space $M$ $=K \bigcup_{a} C L ;$ we described the first method in order to prove this theorem. We then give an application of functional $n$-ary cohomology operations to the general solution of the problem of whether or not $a: L \rightarrow K$ is homotopic to a constant.

Let

$$
0 \rightarrow\left(A^{\prime}, C^{\prime}\right) \stackrel{i}{\rightarrow}(A, C) \stackrel{j}{\rightarrow}\left(A^{\prime \prime}, C^{\prime \prime}\right) \stackrel{k}{\rightarrow}\left(A^{\prime \prime \prime}, C^{\prime \prime \prime}\right) \stackrel{l}{\rightarrow}\left(A^{\prime \prime \prime \prime}, C^{\prime \prime \prime \prime}\right)
$$

be an exact sequence of exact couples. $i, j$, and $k$ are as above and $l$ has bidegree $(0,1)$. Assume further that $\left({ }^{2}\right) \operatorname{Im} k=\operatorname{Ker} l$ in $A^{\prime \prime \prime}, \operatorname{Im} j=\operatorname{Ker} k$ in $A^{\prime \prime}, \operatorname{Im} i=\operatorname{Ker} j$ in $A$, and $k=0$ on $C^{\prime \prime}$. As before, we set $\bar{C}=C / \operatorname{Im} g$. There

(2) Im $k$ is the image of a homomorphism $k$ and Ker $k$ is the kernel of a homomorphism $k$. 
is a natural map $\tau_{n}: C_{n} \rightarrow \bar{C}$. In our application, $\tau_{n}$ will be a monomorphism. Define

$$
\Phi: \operatorname{Ker}\left(i: \bar{C}^{\prime} \rightarrow \bar{C}\right) \rightarrow \operatorname{Ker}\left(l: \bar{C}^{\prime \prime \prime} \rightarrow \bar{C}^{\prime \prime \prime \prime}\right)
$$

of bidegree $(0,0)$, by

$$
\Phi(u)=\left(h^{\prime \prime \prime}\right)^{-1} k\left(f^{\prime \prime}\right)^{-1} j(g)^{-1} i(u) .
$$

It is easy to check that $\Phi$ is well-defined. Note that $\tau_{n}\left(\operatorname{Im} \Delta_{n}\right) \subset \operatorname{Ker}\left(i: \bar{C}^{\prime} \rightarrow \bar{C}\right)$ and $\operatorname{Im} d_{n, k} \subset \operatorname{Ker}\left(l: \bar{C}^{\prime \prime \prime} \rightarrow \bar{C}^{\prime \prime \prime \prime}\right)$.

THEOREM 7.1. $\Phi$ is an isomorphism and $\Phi \tau_{n}^{\prime} \Delta_{n}^{p, q}=d_{n, k}^{p, q}$ when defined $\left({ }^{3}\right)$.

Proof. $\Phi$ is an isomorphism because we may define $\Phi^{-1}$ by $\Phi^{-1}(v)$ $=(i)^{-1} g(j)^{-1} f^{\prime \prime}(k)^{-1} h^{\prime \prime \prime}(v)$ for $v \in \operatorname{Ker}\left(l: \bar{C}^{\prime \prime \prime} \rightarrow \bar{C}^{\prime \prime \prime \prime}\right)$. Furthermore,

$$
\begin{aligned}
\Phi \tau_{n}^{\prime} \Delta_{n}(\{u\}) & =\left(h^{\prime \prime \prime}\right)^{-1} k\left(f^{\prime \prime}\right)^{-1} j(g)^{-1} i(i)^{-1} g(f)^{-n+1} h(j)^{-1}(u) \\
& =\left(h^{\prime \prime \prime}\right)^{-1} k\left(f^{\prime \prime}\right)^{-n} h^{\prime \prime} j(j)^{-1}(u)=d_{n, k}(\{u\}) .
\end{aligned}
$$

The application of this theorem to the two preceding sections is immediate. Hence, we may use either of the two methods of definition of functional $n$-ary cohomology operations. It is to be noted that $\tau_{n}^{\prime}$ is a monomorphism for $p \leqq n$ because $h^{\prime 0, a}$ is onto for all $q$.

Let $a: L \rightarrow K, M=K \bigcup_{a} C L$ as in $\$ 5$. Let $\{u\} \in C_{n}^{p, q}$. Assume that we can define $\Delta_{n}^{p, q} j_{n}(\{u\})$.

THEOREM 7.2. $i_{n} \Delta_{n}^{p, q} j_{n}(\{u\})=d_{n-1}^{p, q}(\{u\}) \in C_{n}^{p+n, q+1}$.

Proof. $i \Delta_{n} j(u)=i(i)^{-1} g(f)^{-n+1} h(j)^{-1} j(u)=g(f)^{-n+1} h(u)=d_{n-1}(\{u\})$.

We now show that functional $n$-ary cohomology operations are sufficient to decide whether or not a given map $a: L \rightarrow K$ is homotopic to a constant when $a$ is in the "stable range."

$a: L \rightarrow K$ is in the stable range if $L$ is a finite dimensional CW-complex, and $K$ is a space of loops. (This definition includes the ordinary stable range because if $\operatorname{dim} L \leqq 2 n-2$ and $K$ is $(n-1)$-connected, then the natural map $\pi(L ; K) \rightarrow \pi\left(L ;{ }^{1} S K\right)$ is an isomorphism.) If $L$ has dimension $N$, we may assume that $\pi_{i}(K)=0$ for $i>N$. We assume that $a^{*}: H^{*}(K ; G) \rightarrow H^{*}(L ; G)$ is zero for all groups $G$ (otherwise, $a$ would not be homotopic to a constant). Let $B$ be the set of higher order cohomology operations coming from the exact couple corresponding to the Postnikov system for $K$.

THEOREM 7.3. $a$ is homotopic to a constant if and only if all functional cohomology operations coming from $a$ and from operations in $B$ are zero.

Proof. The necessity is obvious. Let $M=K \bigcup_{a} C L$ as above. Note that

(3) It is suggested that the reader construct a diagram for the case $n=1$ or 2 to facilitate following this proof. 
$b^{*}: H^{*}(M ; G) \rightarrow H^{*}(K ; G)$ is an epimorphism. Let $I \in \pi(K ; K)=\pi\left(K ; X^{(N+n+2)}\right)$ $=A^{\prime \prime N+2,0}$ denote the identity map, and assume that $K$ is $(n-1)$-connected. To prove sufficiency, we must show that $a^{t}(I)=0$. By exactness, it is enough to show that there is an element $I^{*} \in \pi(M ; K)$ such that $j\left(I^{*}\right)=b^{*}\left(I^{*}\right)=I$. Let $\iota=\left(f^{\prime \prime}\right)^{N+2}(I) \in H^{n}\left(K ; \pi_{n}(K)\right)=C^{\prime \prime 0,0}$. Let $\iota^{*} \in C^{0,0}$ be such that $j\left(\iota^{*}\right)=\iota$. Now $d_{r}^{\prime \prime 0,0}(\iota)=0$ for all $r$, thus, by hypothesis, functional cohomology operations coming from $a$ and from operations in $B$ are zero on $\iota$. By Theorem 7.2, $d_{r}^{0,0}\left(\iota^{*}\right)=0$ and there exists an element $I_{0} \in \pi(M ; K)=A^{N+2,0}$ such that $(f)^{N+2}\left(I_{0}^{i}\right)=\iota^{*}$. Thus $\left(f^{\prime \prime}\right)^{N+2}\left(I-j\left(I_{0}\right)\right)=\iota-\iota=0$, and there is an element $\gamma_{1} \in C^{\prime \prime 1,0}$ such that $h^{\prime \prime}\left(\gamma_{1}\right)=\left(f^{\prime \prime}\right)^{N+1}\left(I-j\left(I_{0}\right)\right)$.

We assume by induction that there is an element $I_{s-1} \in A^{N+2,0}$ and an element $\gamma_{s} \in C^{\prime \prime s, 0}$ such that $h^{\prime \prime}\left(\gamma_{s}\right)=\left(f^{\prime \prime}\right)^{N+2-s}\left(I-j\left(I_{s-1}\right)\right)$. Let $\gamma^{\prime} \in C^{s, 0}$ be such that $j\left(\gamma^{\prime}\right)=\gamma_{s}$. As above, all functional cohomology operations vanish on $\gamma_{s}$ and hence $d_{r}^{s, 0}\left(\gamma^{\prime}\right)=0$ for all $r$. Thus there is an element $I^{\prime} \in A^{N+2,0}$ such that $(f)^{N+2-s}\left(I^{\prime}\right)=h\left(\gamma^{\prime}\right)$. Define $I_{s}=I_{s-1}-I^{\prime}$. Then $\left(f^{\prime \prime}\right)^{N+2-s}\left(I-j\left(I_{s}\right)\right)$ $=\left(f^{\prime \prime}\right)^{N+2-s}\left(I-j\left(I_{s-1}\right)-j\left(I^{\prime}\right)\right)=h^{\prime \prime}\left(\gamma_{s}\right)-j(f)^{N+2-s}\left(I^{\prime}\right)=h^{\prime \prime}\left(\gamma_{s}\right)-j h\left(\gamma^{\prime}\right)=0$. Thus there is an element $\gamma_{s+1} \in C^{\prime \prime s+1,0}$ such that $h^{\prime \prime}\left(\gamma_{s+1}\right)=\left(f^{\prime \prime}\right)^{N+2-s-1}\left(I-j\left(I_{s}\right)\right)$. This completes the induction step. To complete the proof, put $I^{*}=I_{N+1}$, and note that $\gamma_{N+2} \in C^{\prime \prime N+2,0}=0$, because $\pi_{i}(K)=0$ for $i>N$. Thus $I=j\left(I^{*}\right)$.

8. The composition theorem. We now prove one of our main theorems. This theorem gives sufficient conditions for the composition of two nontrivial maps to be non trivial. It shows that under certain conditions, the composition of a functional $m$-ary cohomology operation and a functional $n$-ary cohomology operation is a functional $(m+n)$-ary cohomology operation.

Let $a: L \rightarrow K$ and $b: M \rightarrow L$. Then we have functional cohomology operations defined by $a^{*}$, denoted by $d_{n, a}^{p, q}$, and defined by $b^{*}$, denoted by $d_{n, b}^{p, q}$.

THEOREM 8.1. When both sides are defined, $d_{m+n, a b}^{p, q}=d_{n, b}^{p+m, q} d_{m, a}^{p, q}$.

Proof. The proof, as the statement of the theorem, will be given in the algebraic language of the second method. Let $(A, C)$ be the exact couple for $K$ (corresponding to a given Postnikov system), $\left(A^{\prime}, C^{\prime}\right)$ for $L$, and $\left(A^{\prime \prime}, C^{\prime \prime}\right)$ for $M$. Let $\{u\} \in C_{m+n}^{p, q}$. Let $v \in A^{p+m+n, q}$ be a lifting of $u(m+n)$-stages. Then $a^{\sharp}(v)$ is a lifting of $d_{m, a}^{p, q}(\{u\}) n$-stages. Hence $d_{m+n, a b}^{p, q}(\{u\})=\left(h^{\prime p+m+n, q}\right)^{-1}(a b)^{\sharp}(v)$ $=\left(h^{\prime \prime p+m+n, q}\right)^{-1} b^{\sharp}\left(a^{\#}(v)\right)=d_{n, b}^{p+m, q} d_{m, a}^{p, q}(\{u\})$.

9. Some applications of the composition theorem. Let $a: L \rightarrow K$ and $b: M \rightarrow L$ be two maps. If $a$ and $b$ are both nontrivial, then is the composition $a b$ nontrivial? In this section, we describe how Theorem 8.1 gives sufficient conditions for $a b$ to be nontrivial, and we also give some examples of this technique.

Assume that $a$ is shown to be nontrivial by a functional $m$-ary cohomology operation $d_{m, a}^{p, q}$ and that $b$ is shown to be nontrivial by a functional $n$-ary cohomology operation $d_{n, b}^{\prime p, q}$. In such a situation, it is obvious from Theorem 8.1, that if we can construct a Postnikov system such that the corresponding 
exact couple contains the $m$-ary cohomology operation associated to $d_{m, a}^{p, q}$ and the $n$-ary cohomology operation associated to $d_{n, b}^{p, q}$ at the level $(p+m, q)$, then $a b$ is nontrivial. In general, we do not have the technique to construct such Postnikov systems. One important difficulty arises from the fact that we cannot compute Steenrod squares and reduced $p$ th powers in a space having two nonvanishing homotopy groups (see appendix). Such problems as $[9$, p. 337] should be amenable to this technique.

Let us conclude by giving a couple of examples where the computations needed to apply Theorem 8.1 can be made. Let $b: S^{n+2} \rightarrow S^{n+1}$ and $a: S^{n+1} \rightarrow S^{n}$ be essential maps. Let $\theta=S q^{2}: H^{n}(K ; Z) \rightarrow H^{n+2}\left(K ; Z_{2}\right)$. Then there is a $\theta^{\prime}: X \rightarrow K\left(Z_{2} ; n+3\right)$ such that $\left(\theta^{\prime} \omega\right)^{*}: H^{n+1}\left(K ; Z_{2}\right) \rightarrow H^{n+3}\left(K ; Z_{2}\right)$ is also $S q^{2}$ (this is due to the fact that $S q^{2} S q^{2}=S q^{3} S q^{1}=0$ on $H^{n}(K ; Z)$ ). Because $a$ and $b$ are nontrivial, $d_{1, a}^{0,0}=S q_{a}^{2}: H^{n}\left(S^{n} ; Z\right) \rightarrow H^{n+1}\left(S^{n+1} ; Z_{2}\right)$ and $d_{1, b}^{1,0}=S q_{b}^{2}$ : $H^{n+1}\left(S^{n+1} ; Z_{2}\right) \rightarrow H^{n+2}\left(S^{n+2} ; Z_{2}\right)$ are both nonzero (this follows from Theorem 7.2 and $[1$, p. 724$])$. Hence by Theorem $8.1, d_{2, a b}^{0,0}: H^{n}\left(S^{n} ; Z\right) \rightarrow H^{n+2}\left(S^{n+2} ; Z_{2}\right)$ is nonzero and hence $a b$ is not homotopic to a constant. Notice that in our case, the conditions needed to define all of our operations are trivially satisfied and there are no subgroups to factor out. This is a well-known result of Adem [1, p. 723]; his other results on compositions of maps of Hopf invariant one can be proven similarly by our technique.

Our second example generalizes the above theorem modulo $p$. First, we make an obvious comment. It is known that $\left({ }^{4}\right)$

$$
\pi_{r}\left(S^{n}\right)_{p}= \begin{cases}0 & \text { if } r<n+2 p-3 \text { or } n+2 p-3<r<n+4 p-5, \\ Z_{p} & \text { if } r=n+2 p-3 \text { or } r=n+4 p-5\end{cases}
$$

(we assume throughout that we are in the stable range). Hence if $a: S^{n+2 p-3}$ $\rightarrow S^{n}$ represents a generator of $\pi_{n+2 p-3}\left(S^{n}\right)_{p}$ and $b: S^{n+4 p-6} \rightarrow S^{n+2 p-3}$ represents a generator of $\pi_{n+4 p-6}\left(S^{n+2 p-3}\right)_{p}$, then $a b$ is homotopic to a constant by dimensional reasons. Thus our generalization will have to take a different form.

A map $a: S^{r} \rightarrow S^{n}$ is said to have $\bmod p H o p f$ invariant one if $[a] \in \pi_{r}\left(S^{n}\right)$ has order a power of $p$ and if $a$ is shown to be nontrivial by a functional reduced $p$ th power of Steenrod. It follows immediately from the Adem basis theorem [2] and Theorem 7.2, that if $a$ has $\bmod p$ Hopf invariant one, then $r-n=2 p^{k}(p-1)-1$ for some $k$. It is known that there exist maps of $\bmod p$ Hopf invariant one in case $k=0$ or 1 ([5] and [11]). ${ }^{(5)}$

Let $a: S^{n+2 p l(p-1)-1} \rightarrow S^{n}$ be a map of $\bmod p$ Hopf invariant one, and let [a] have order $p^{s}$. Let $X=S^{n+2 p l(p-1)-1} \cup e^{n+2 p l(p-1)}$, where $e^{n+2 p l(p-1)}$ is attached by a map of degree $p^{s}$. Then we can obviously extend $a$ to a map

(4) $A_{p}$ denotes the $p$-primary component of the abelian group $A$.

(5) Added in proof. Recently, Borel and others have shown that no map of $\bmod p$ Hopf invariant one exists if $k=1$. Furthermore, the computations made in the following two paragraphs are correct only in the case $k=1=0$. The error is in the fact that $\delta^{*}$ is 0 in the space $X$ if $s>1$. 
$\bar{a}: X \rightarrow S^{n} . \bar{a}$ is also shown to be nontrivial by a functional $\rho^{p l}$ by Theorem 6.1 . Let $b: S^{n+2\left(p l+p^{k}\right)(p-1)-1} \rightarrow S^{n+2 p l(p-1)}$ be another map of mod $p$ Hopf invariant one. Using Theorem 3.6 of $\left[13\right.$, p. 264], setting $K=S^{n+2(p l+p k)(p-1)-1}$, $r=n+2 p^{l}(p-1)-1$, and $G=Z_{p^{*}}$, we get a map $b: S^{n+2\left(p^{l}+p^{k}\right)(p-1)-1} \rightarrow X$ such that $b$ followed by a given map $X \rightarrow S^{n+2 p^{l}(p-1)}$ is homotopic to $b . b$ is shown to be nontrivial by a functional $\rho^{p^{k}} \delta^{*}$ by Theorem 6.2. We now show that if $l \geqq k$, then $\bar{a} b: S^{n+2\left(p^{\left.l+p^{k}\right)(p-1)-1} \rightarrow S^{n}\right.}$ is nontrivial( $\left.{ }^{6}\right)$.

Our problem reduces to constructing a Postnikov system with $d_{m}^{0,0}=\rho^{p l}$ and $d_{q}^{m, 0}=P^{p^{k}} \delta^{*}$, for then we may apply Theorem 8.1 as in the first example. Start with $K(Z, n)$, and use coefficients $Z_{p}$ throughout. Let $\iota$ be the canonical element of $H^{n}\left(Z, n ; Z_{p}\right)$. Construct $X, X^{\prime}, \cdots, X^{(m-1)}$ by successively killing $\rho^{1}(\iota), \rho^{2}\left(\iota_{1}\right), \cdots, \rho^{p-1}\left(\iota_{m-1}\right)$, where $m=p^{k-1}$. Now construct $X^{(m)}$ with $k$-invariant $\rho^{p l}\left(\iota_{m}\right) .\left(\iota_{j}\right.$ is the image of $\iota$ in $X^{(j-1)}$.) If $l>k$, then $\rho^{p^{k}} \delta^{*} \rho^{p l}\left(\iota_{m+1}\right)$ $=0$, and thus there is a $\theta^{(m+1)} \in H^{n+2\left(p l+p^{k}\right)(p-1)}\left(X^{(m)} ; Z_{p}\right)$ such that $\left(\theta^{(m+1)} \omega^{(m)}\right)^{*}=\rho^{p k} \delta^{*}$. This follows from the Adem relations [2], our constructions, and the fact $C\left(p^{l}(p-1), p^{k}\right) \equiv 0 \bmod p$, where $C(m, n)$ is the binomial coefficient $\left(\begin{array}{c}m \\ n\end{array}\right)$. If $l=k$, then

$$
\left(\rho^{p^{k}} \delta^{*}-C\left(p^{k}(p-1)-p, p^{k}\right)^{-1} C\left(p^{k}(p-1), p^{k}\right) \delta^{*} \rho^{p k}\right) \rho^{p^{k}}\left(\iota_{m+1}\right)=0,
$$

and thus there is a $\theta^{(m+1)} \in H^{n+4 p^{k}(p-1)}\left(X^{(m)} ; Z_{p}\right)$ such that

$$
\left(\theta^{(m+1)} \omega^{(m)}\right)^{*} \rho^{p^{k}} \delta^{*}-C\left(p^{k}(p-1)-1, p^{k}\right)^{-1} C\left(p^{k}(p-1), p^{k}\right) \delta^{*} \rho^{p^{k}} .
$$

This follows similarly to the above because $C\left(p^{k}(p-1)-1, p^{k}\right) \not \equiv 0 \bmod p$.

\section{APPENDIX}

10. Cohomology of fibre spaces. We conclude this paper with some remarks designed to facilitate understanding the computations of $\$ 9$.

Let $p: E \rightarrow B$ be a fibre space with fibre $F$. Associated to this fibre space, there is a spectral sequence with $E_{2}^{p, q} \approx H^{p}\left(B ; H^{q}(F)\right)$ and $E_{\infty}$ the graded group associated with $H^{*}(E)[15$, p. 451]. Assume, as is the case in $\S 9$, that we have a field of coefficients and that we are working in the stable range; i.e. we consider $H^{n}(E)$ where $n \leqq r+s, B$ is $r$-connected, and $F$ is $s$-connected. Elements of $H^{n}(E)$ arise in two ways; i.e. $H^{n}(E)=E_{\infty}^{n, 0}+E_{\infty}^{0, n} . E_{\infty}^{n, 0} \approx p^{*}\left(H^{n}(B)\right.$ $\approx H^{n}(B) / d_{n}^{0, n-1}\left(H^{n-1}(F)\right) . E_{\infty}^{0, n} \approx \operatorname{Ker}\left(d_{n+1}^{0, n}: H^{n}(F) \rightarrow H^{n+1}(B)\right)$. A fundamental problem is how does one compute squares or reduced powers in $H^{*}(E)$ if they are known in $H^{*}(F)$ and in $H^{*}(B)$ ? For elements in $E_{\infty}^{n, 0}$, the answer is easy. Since squares or reduced powers commute with $p^{*}$, the computation can be carried out in $H^{*}(B)$; this type of computation was used in the second example of $\$ 9$. However, $E_{\infty}^{0, n}$ is not naturally imbedded in $H^{n}(E)$, and thus the computation of squares or reduced powers of elements in $E^{0, n}$ is much more difficult. It is this type of computation that will lead to deeper results.

${ }^{6}$ ) Adem [3] proves the case $k=l=0$ and some other results of a similar nature by a different technique. 


\section{BibliogRAPHY}

1. J. Adem, The iteration of Steenrod squares in algebraic topology, Proc. Nat. Acad. Sci. U.S.A. vol. 38 (1952) pp. 720-726.

2. - Relations on iterated reduced powers, Proc. Nat. Acad. Sci. U. S. A. vol. 39 (1953) pp. 636-638.

3. - Un criterio cohomológico para determinar composiciones esenciales de transformaciones, Boletín de la Sociedad Matemática Mexicana, II, vol. 1 (1956) pp. 38-48.

4. M. G. Barratt, Track groups I, Proc. London Math. Soc. (3) vol. 5 (1955) pp. 71-106.

5. A. Borel and J.-P. Serre, Groupes de Lie et puissances réduites de Steenrod, Amer. J. Math. vol. 75 (1953) pp. 409-448.

6. H. Cartan and S. Eilenberg, Homological algebra, Princeton University Press, 1956.

7. A. Heller, Homotopy resolutions of semi-simplicial complexes, Trans. Amer. Math. Soc. vol. 80 (1955) pp. 299-344.

8. - Exact couples in algebraic topology, I and II, III, IV, and V, Ann. of Math. vol. 56 (1952) pp. 363-396; vol. 57 (1953) pp. 248-286.

9. - Some problems in algebraic topology and the theory of fibre bundles, Ann. of Math. vol. 62 (1955) pp. 327-359.

10. W. S. Massey, Some higher order cohomology operations, Proceedings of the International Symposium on Algebraic Topology, Mexico City, 1956.

11. J. C. Moore, The double suspension and p-primary components of homotopy groups of spheres, Boletín de la Sociedad Matemática Mexicana (II) vol. 1 (1956) pp. 28-37.

12. F. P. Peterson, Some results on cohomotopy groups, Amer. J. Math. vol. 78 (1956) pp. 243-258.

13. - Generalized cohomotopy groups, Amer. J. Math. vol. 78 (1956) pp. 259-282.

14. M. M. Postnikov, Investigations in homotopy theory of continuous mappings, Trudy Mat. Inst. Steklov. no. 46. Izdat. Akad. Nauk SSSR. Moscow, 1955 (in Russian).

15. J.-P. Serre, Homologie singulière des espaces fibrés, Ann. of Math. vol. 54 (1951) pp. 425-505.

16. - Cohomologie modulo 2 des complexes d'Eilenberg-MacLane, Comment. Math. Helv. vol. 27 (1953) pp. 198-231.

17. M. Shimada, Homotopy classification of mappings of a 4-dimensional complex into a 2-dimensional sphere, Nagoya Mathematical Journal vol. 5 (1953) pp. 127-144.

18. E. H. Spanier and J. H. C. Whitehead, A first approximation to homotopy theory, Proc. Nat. Acad. Sci. U. S. A. vol. 39 (1953) pp. 655-660.

19. N. E. Steenrod, Cohomology invariants of mappings, Ann. of Math. vol. 50 (1949) pp. 954-988.

20. H. Suzuki, On the Eilenberg-MacLane invariants of loop spaces, Journal of the Mathematical Society of Japan vol. 8 (1956) pp. 40-48.

University of Chicago, Chicago, Ill. 